\title{
TRENDS IN SEXUAL BEHAVIOUR IN CZECH SCHOOLCHILDREN BETWEEN 2002-2014
}

\author{
Zlatko Pastor ${ }^{1,2,3}$, Petr Weiss ${ }^{2}$, Dagmar Sigmundová ${ }^{4}$ \\ 'Department of Obstetrics and Gynaecology, Second Faculty of Medicine, Charles University and Motol University Hospital, Prague, Czech \\ Republic \\ ${ }^{2}$ Institute of Sexuology, First Faculty of Medicine, Charles University, Prague, Czech Republic \\ ${ }^{3}$ National Institute of Mental Health, Klecany, Czech Republic \\ ${ }^{4}$ Institute of Active Lifestyle, Faculty of Physical Culture, Palacký University Olomouc, Olomouc, Czech Republic
}

\begin{abstract}
SUMMARY
Objective: The aim of this study was to report on trends in the prevalence in age at first intercourse of Czech schoolchildren and a difference between sexes.

Methods: Prevalence in age at first intercourse of Czech schoolchildren was observed under the Health Behaviour in School-aged Children study in the years 2002, 2006, 2010 and 2014. In addition, a random representative sample of children attending 9th grade completed the questions related to sexual behaviour. A total of 4,804 boys and girls at the age of 15 years were included in the study. The chi-square test and analysis of variance were used for data assessment.

Results: Experience with first sexual intercourse before 15 years was confirmed by $16 \%$ of boys and $19.4 \%$ of girls. Average age of coitarche in both sexes most often ranged between 14 and 15 years. Since 2006 boys had significantly lower age at coitarche. Only in 2010 the girls had first intercourse more frequently than boys. Since 2010 the number of Czech adolescents of both sexes having first intercourse before the age of 15 years has significantly increased. However, a rise in average age of coitarche over 15 years in both sexes occurred in 2014.

Conclusions: The number of Czech children having a sexual intercourse before 15 years has significantly increased since 2010.
\end{abstract}

Key words: age at first sexual intercourse, coitarche, schoolchildren

Address for correspondence: Z. Pastor, Department of Obstetrics and Gynaecology, Second Faculty of Medicine, Charles University and Motol University Hospital, V Úvalu 84, 15000 Prague 5, Czech Republic. E-mail: pastor.zlatko@volny.cz

https://doi.org/10.21101/cejph.a4821

\section{INTRODUCTION}

First sexual intercourse is considered to be a major life event, which also may have significant health and social implications. Circumstances of initiation of sexual life are one of main indicators of human sexual behaviour. Beginning of sexual activity may have a decisive impact on subsequent sexual activities of an individual, but also on other psychosocial aspects. In the medical literature on sexual behaviour, a primary focus has been on the debut of sexual intercourse because earlier initiation of sex is powerful predictor of HIV risk behaviour, sexuality transmitted infections, as well as early unintended pregnancy (1).

One of the strongest correlates of early sex is substance use (2). Correlation between early sexual onset and more frequent use of addictive substances were well documented in the systematic review of Zimmer-Gembeck and Helfand, which compared 35 studies on this topic (3). Factors related to early age at sexual intercourse and increased risk of genital infections have been linked to higher prevalence of neoplastic diseases in genito-anal area, in particular carcinomas of cervix in women and rectal cancer in homosexual men (4). Factors which are associated with sexual debut before the age of 15 years lead to poorer psychosocial and risk-taking behaviour in later life and are observed in most of the surveys of sexual activities in various countries. A summary of these researches from 14 countries is provided in the paper of Singh et al. (5) or in the study covering 17 European countries (6).

Weiss and Zvěřina observed in the last decade declining trend of age at first intercourse in population in the Czech Republic, which corresponds to the conclusions of Máthé and Ritomský reporting a change from 18 to $15-17$ years of coitarche in adolescents in Slovakia in $2004(7,8)$. Kastbom et al. confirm the association between start of sexual life before 14 years of age and various socio-demographic indicators, which are causes of more frequent sexual experiences, health problems, drug use, child or sexual abuse. Early sexual initiation is positively linked to the number of partners, experience of oral and anal sex, smoking, drug and alcohol use, and other risky behaviour as lying or stealing. The risk of psychiatric symptoms, low self-esteem or low sense of coherence were not associated to early commencement of sexual life (9). From earlier but mostly representative researches with similar conclusion we may mention British study of Johnson et al. (10), US study of Michael et al. (11), Bingham et al. (12), Canadian paper of Odynak (13), or French study of Spira and Bajos (14).

Most recent studies observe trends in initiation of sexual life in connection with changes in the lifestyle of contemporary 
children. Safeguarding Teenage Intimate Relationships (STIR) is a research project funded by the Daphne III Programme of the European Commission's Directorate General Justice. It is a 2-year project in which 4,564 young people of 13 and 17 years of age from Bulgaria, Cyprus, England, Italy, and Norway took part. One of the aims was to explore impact of new technologies (mobiles, social networks, chats etc.) on sexual relationships of teenagers. This multicentral study reports that most (72\%) of these children aged 14-17 years had already a sexual partner, between 6 and $44 \%$ of young women and between 15 and $32 \%$ of boys had ever sent or received sexual image or text message. Between 13 to $59 \%$ of female adolescents and 9 to $25 \%$ of male adolescents said that it had negative impact on them (15).

Early initiation of sexual life can also be related to insufficient or low quality sexual education in a family or in educational system. In the study of NUS (National Union of Students) in the UK, in which 2,500 students took part and half of them were aged from 16 to 19 years, reported that they had watched porn to better understand sex and because of a lack of adequate education they consider these sources as relevant information on sexuality (16). The aim of our longitudinal research was to find basic information about age at first intercourse of Czech boys and girls and to examine whether there is a difference between sexes.

We focused on observations of trends in prevalence in age at first intercourse so that we can evaluate development of these factors in the cycles in 2002, 2006, 2010 and 2014, and to use it to comment on the trends and changes in sexual behaviour of Czech adolescents.

\section{MATERIALS AND METHODS}

Data from the Czech sample of the Health Behaviour in School-aged Children study (HBSC) were collected using a selfadministered questionnaire. School-based anonymous surveys were conducted in 2002, 2006, 2010 and 2014 according to a common research protocol (17). A national random representative sample of children attending 9th grade was selected to complete the questions related to sexual behaviour. The completion of the HBSC questionnaire by children was voluntary. The final data inspection was performed by the International Databank, University of Bergen, Norway, in compliance with the international HBSC standards. Dataset for this analysis included 1,350 children (48\% of boys, $16 \%$ missing values) in $2002,1,439$ children $(51 \%$ of boys, $20 \%$ missing values) in 2006, 1,370 children ( $48 \%$ of boys, $25 \%$ missing values) in 2010 and 1,539 children ( $50 \%$ of boys, $4 \%$ missing values) in 2014 . The findings below are based on data collected from 4,804 children aged 15-15.99 years. In 2002-2014 surveys, two items on the sexual behaviour were used. Adolescents were asked:

- Have you ever had sexual intercourse (sometimes this is called "making love," "having sex," or "hooking up", "sleeping with someone", "making out", or "doing it")? The response options were "yes" or "no".

- How old were you when you had sexual intercourse for the first time? For this question, the response options were "11 years old or younger", "12 years old", "13 years old", "14 years old", "15 years old", "16 years old or older".

\section{Statistical Analysis}

To assess differences in the prevalence of sexual intercourse between boys and girls, and between years of survey, chi-square test was used. Differences in the age of first sexual intercourse between survey years $(2002,2006,2010,2014)$ or between boys and girls were computed according to Univariate analysis of variance with given LSD post hoc test (Levene's test for homogeneity of variances, $\mathrm{p}_{\text {boys }}=0.357 ; \mathrm{p}_{\text {girls }}=0.223$ ).

\section{RESULTS}

For this study, we asked 5,698 respondents whether they had first sexual intercourse, with a response rate of $84.3 \%(4,804)$. In total $894(15.6 \%)$ respondents did not reply, out of which $18.2 \%$ were boys and $13.2 \%$ girls. Experience with first sexual intercourse before 15 years was confirmed by $16 \%$ of boys and $19.4 \%$ of girls. $7.8 \%$ of boys and $6.9 \%$ of girls had coitarche in the age of 14 years or less. The question about first sexual intercourse was given only to children from the age category 15-15.9 years. The term "sexual intercourse" was chosen instead of "vaginal intercourse" as a synonym which is commonly used in this age group for genitogenital intercourse. Average age of first intercourse in both sexes most often ranged between 14 and 15 years. Based on obtained data, in 2002 it was 17\% for both the boys and the girls who had first sexual intercourse, in $200616 \%$ of boys and $19 \%$ of girls, in $201021 \%$ of boys and $27 \%$ of girls, and in 2014 it was $23 \%$ of boys and $26 \%$ of girls. Percentage ratio of girls with first sexual intercourse before 15 years was always higher in comparison to boys but significant difference was observed only in 2010 when the girls with experienced vaginal intercourse unequivocally outnumbered boys (chi-square test 4.776, significance level 0.029) (Table 1). Based on the chi-square test, prevalence of sexual intercourse in girls before 15 years differed among the cycles at the following significance levels: 2002 and $2010(p<0.001), 2002$ and $2014(\mathrm{p}<0.001), 2006$ and $2010(\mathrm{p}=0.006)$ and 2006 and 2014 $(p=0.003)$. Prevalence of first intercourse in boys before 15 years in the surveyed period differed: 2002 and $2014(p=0.005), 2006$ and $2010(p=0.025), 2006$ and $2014(p=0.001)$.

Significant increase of prevalence of first sexual intercourse in both sexes occurred in 2010 and this trend continued also in 2014 (Table 2).

Based on the ANOVA test, significantly lower age of first sexual intercourse in boys than in girls was seen in the following years: $2002(\mathrm{~F}=5.07, \mathrm{p}=0.026), 2010(\mathrm{~F}=16.33, \mathrm{p}<0.001)$ and in $2014(\mathrm{~F}=10.20, \mathrm{p}=0.002)$ (Fig. 1.).

Our research shows that just under one fifth of our respondents ( $16 \%$ of girls and $19 \%$ of boys) had first sexual intercourse before 15 years. No significant difference in age at first intercourse was seen with exception of 2010 when girls had first intercourse more frequently. Since 2006 boys have significantly lower age at coitarche. The proportion of adolescents having first sexual intercourse before 15 years substantially increased in both sexes from 2010 .

\section{DISCUSSION}

From sexological-legal point of view, the boys and the girls who had sexual intercourse before the age of consent (which is 
Table 1. Gender differences in prevalence of first sexual intercourse in 15 years old children in 2002, 2006, 2010 and 2014

\begin{tabular}{|c|c|c|c|c|}
\hline Survey year & Gender & $\begin{array}{l}\text { Prevalence of sexual } \\
\text { intercourse }\end{array}$ & $x^{2}$ & $p$ \\
\hline \multirow{2}{*}{2002} & Boys & $90(17 \%)$ & \multirow{2}{*}{0.003} & \multirow{2}{*}{0.95} \\
\hline & Girls & $106(17 \%)$ & & \\
\hline \multirow{2}{*}{2006} & Boys & $91(16 \%)$ & \multirow{2}{*}{1.448} & \multirow{2}{*}{0.229} \\
\hline & Girls & $112(19 \%)$ & & \\
\hline \multirow{2}{*}{2010} & Boys & $97(21 \%)$ & \multirow{2}{*}{4.776} & \multirow{2}{*}{0.029} \\
\hline & Girls & $148(27 \%)$ & & \\
\hline \multirow{2}{*}{2014} & Boys & $170(23 \%)$ & \multirow{2}{*}{1.806} & \multirow{2}{*}{0.179} \\
\hline & Girls & $195(26 \%)$ & & \\
\hline
\end{tabular}

${ }^{*} \mathrm{~N}$ number (\% proportion) of boys and girls who answered that they had sexual intercourse, $\mathrm{X}^{2}$ chisquare test, $\mathrm{p}$ - statistical significance

Table 2. Trends in prevalence of sexual intercourse: HBSC study 2002-2014

\begin{tabular}{|c|c|c|c|c|c|c|c|c|c|c|c|c|c|}
\hline \multirow{3}{*}{ Variable } & \multirow{3}{*}{$\begin{array}{c}2002 \\
\%\end{array}$} & \multirow{3}{*}{$\begin{array}{c}2006 \\
\%\end{array}$} & \multirow{3}{*}{$\begin{array}{c}2010 \\
\%\end{array}$} & \multirow{3}{*}{$\begin{array}{c}2014 \\
\%\end{array}$} & \multicolumn{3}{|c|}{2006 vs. 2002} & \multicolumn{3}{|c|}{2010 vs. 2002} & \multicolumn{3}{|c|}{2014 vs. 2002} \\
\hline & & & & & \multirow{2}{*}{ OR } & \multicolumn{2}{|c|}{$\mathrm{Cl}$} & \multirow{2}{*}{ OR } & \multicolumn{2}{|c|}{$\mathrm{Cl}$} & \multirow{2}{*}{ OR } & \multicolumn{2}{|c|}{$\mathrm{Cl}$} \\
\hline & & & & & & Lower & Upper & & Lower & Upper & & Lower & Upper \\
\hline \multicolumn{14}{|l|}{ Boys } \\
\hline $15-15.99$ years & 17.2 & 16.2 & 20.7 & 23.1 & 0.93 & 0.68 & 1.28 & 1.26 & 0.9178 & 1.734 & $1.45^{*}$ & 1.09 & 1.92 \\
\hline \multicolumn{14}{|l|}{ Girls } \\
\hline $15-15.99$ years & 17.3 & 18.9 & 26.6 & 26.1 & 1.11 & 0.83 & 1.49 & $1.73^{\star \star \star}$ & 1.31 & 2.29 & $1.69^{\star * *}$ & 1.29 & 2.20 \\
\hline
\end{tabular}

Results from logistic regression (method Enter); \% percentage of participants who had sexual intercourse; OR: odds ratio (cohorts from the year 2002 are reference groups); Cl: $95 \%$ confidence interval; ${ }^{*} p \leq 0.05 ;{ }^{* *} p \leq 0.01 ;{ }^{* *} p \leq 0.001$

the age until which a child is protected from early sexualisation in civilised countries and which is 15 years in the Czech Republic), can be considered as victims of sexual abuse. The conclusions of Wyatt (18), Jeffrey and Jeffrey (19), Rogers and Terry (20), and Gianotten (21) point out differences between abused and nonabused men and women in a partnership and sexual adaptation, in marital and parental roles, with higher prevalence of sexual, social and mental pathology among abused persons.

A consensual intercourse between individuals up to 15 years, according to Czech law, is considered in other softer mode than sexual abuse of a child by a person older than 15 years. Even though the sexual activities of two children do not have to have a character of a trauma, it can have adverse consequences for subsequent psychosexual and personal development. Even if

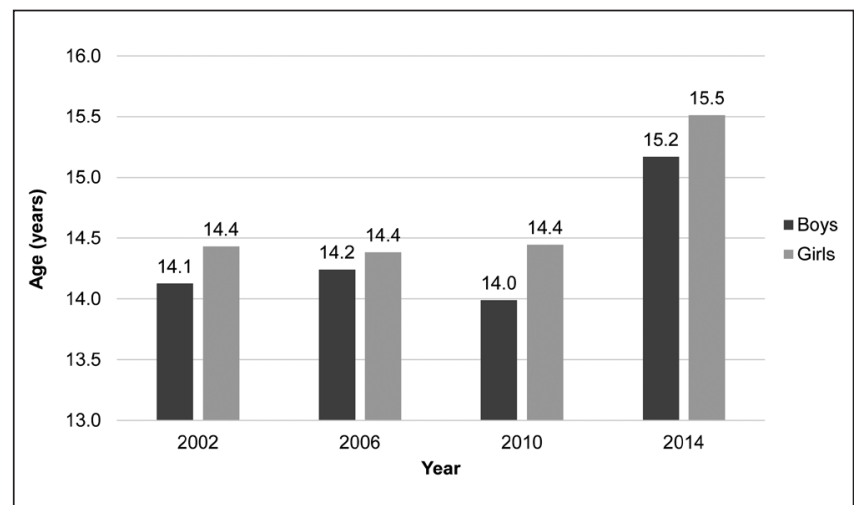

Fig. 1. Mean age at first sexual intercourse in 2002, 2006, 2010 and 2014 coitarche does not occur as a form of abuse by adult offender (man or woman), but for instance as a sexual experiment or agreement between similarly aged partners, lower age of first intercourse according to the above-mentioned studies is linked to many other risk factors and it has unfavourable impact on later development of respondents.

According to Lukšík (22) individuals who had first intercourse before 16 years, have statistically significantly higher risk in further sexual life, for instance they have higher number of sexual partners, more casual partners and they show more frequent consumption of addictive psychoactive substances. It is essential, from the above reasons, to determine which factors are important for early sexualisation of children. Many authors pursue definition of these factors. For instance, Gibbs (23) writes about impact of a change in social norms regarding the sex life or influence of media on adolescents. It is reported that decline in age at coitarche in the US is impacted also by the fact that a teenager watching TV programs in average five hours, can see about 14,000 of intercourses yearly.

Some authors point out that coitus is initiated by the boys and girls from incomplete or complemented families (i.e. they are raised by a step parent) and children of parents with lower socieconomical status (lower education and less qualified and less valued occupation). They are much more risky not only because of early onset of sexual activity but also with respect to broader spectrum of potential threat. Children raised by step parents have, compared to children from complete families, many times higher likelihood that they would be a victim of not only sexual abuse, but traffic accident or suicide (24). Evidence about negative impact of incomplete family on early initiation of sexual life is 
presented in the research of Gibbs (23), Voydanoff and Donnelly (25), and Larson et al. (26). Zimmerman and Atwood (27) in their large cohort of 1,131 adolescents evidence that completeness of original family is essential for a cut of early sex initiation risk. One of recent national American studies of Jordahl and Lohman (28) proved that $13 \%$ of girls and $15 \%$ of boys had sex before 16 years. According to the authors, the proportions of sexually experienced girls coming from the families with various economical levels are not different, but in boys almost twice as much are from the families with low socioeconomical status. Their conclusion is that low income and family instability are risky factors for early sexual activity. Similarly, Bingham et al. (12) found a correlation between lower economical family status and more frequent coitarche in American teenage girls.

The results of our research, focused on the number of children having a sexual intercourse before 15 years, partially correspond to the results of similar foreign studies (16\% of boys, $19 \%$ of girls in our study versus $13 \%$ of girls and $15 \%$ of boys in the paper of Jordahl and Lohman (28). Swedish research from 2015 reports that $7.5 \%$ of students had coitarche before 14 years $(7.8 \%$ of girls and $7.2 \%$ of boys), which responds to the results of our study where $7.8 \%$ boys and $6.9 \%$ of girls had sexual intercourse before 14 years (9). However, our study did not contain any socioeconomical status and family structure information about our respondents, so in this sense the results cannot be compared. Our focus was mainly on comparison of differences between both sexes, whether coitarche before 15 years is more frequent in girls or boys, which was not confirmed in most of the cases. The age at first intercourse before 15 years and its trends were other observed facts. An increasing trend from 2010 has been evidenced, when the number of adolescents of both sexes having first sexual intercourse before 15 years has been rising. The results of this trend correspond to other national researches of sexual behaviour which report that from the 1960's the age at first coitus in Western Europe has decreased and more among women than men.

According to HBSC study from 1997-98 realized in seven European countries, around $30 \%$ of boys had coitarche before 15 years. $47 \%$ of boys had first sexual intercourse at this age in Hungary. The study shows that significantly more girls than boys had coitarche before 15 years, in Scotland 37\%, in Hungary 34\% and in Finland 30\% (29). British study from 2013 involving over 15,000 adults aged 16-74 reports that median age at first heterosexual intercourse was 17 years in both sexes but it was 16 years in those aged 16-24 years at interview, which confirms the fact of declining age at coitarche. Among this age group, the latest survey found that $31 \%$ of men and $29 \%$ of women now have first sex before the age of 16 in contrast to the group of 55-64 years old in which $17 \%$ of men and $10 \%$ of women had intercourse before 16th birthday (30). The upward trend in the proportion of adolescents with coitarche before 15 years cannot be clearly explained. We can only assume that it can be associated with secondary sexual acceleration, rapid somatosexual maturation, generally higher erotisation of society, a larger number of evident and subliminal sexual stimuli on young people, use of the Internet and various online social networks, inadequate sexual education in family and institutions, influence of media, secular society in our country, and other social factors. It is not evident whether this trend is the breaking point which will further continue, stagnate or will have opposite tendency or by which factors is mostly affected. Future studies investigating circumstances, incentives and trends of early initiation of sexual life are required to clarify all the above-mentioned questions.

\section{Strengths and Limitations}

A study on a large sample of Czech children in the context of longitudinal observation of long time period could competently assess trends of first sexual intercourse in monitored age category. However, the described research was not primarily designed as a sexology study and therefore, with exception for first sexual intercourse, we cannot comment on other sexological aspects and its trends such as the number of partners, character of sexual activities, contraceptive behaviour, and others. From similar reason, we do not discuss the relationship and circumstances of first sexual intercourse with other socioeconomical factors, which were not part of the trend analysis.

\section{CONCLUSION}

Based on obtained results we can state that trends in the prevalence of first intercourse in Czech male and female adolescents before 15 years show an increasing proportions in both sexes from 2010. Future research will confirm whether this is just a random fact or it is a beginning of increase in average age at first sexual intercourse.

\section{Acknowledgements}

This work was supported by the research grant of the Czech Science Foundation Reg. No. 17-12579S and by the Czech Ministry of Education, Youth, and Sports (MEYS) under Contract No. LG 14043.

\section{Conflict of Interests}

None declared

\section{REFERENCES}

1. Bachanas PJ, Morris MK, Lewis-Gess JK, Sarett-Cuasay EJ, Flores AL Sirl KS, et al. Psychological adjustment, substance use, HIV knowledge, and risky sexual behavior in at-risk minority females: developmental differences during adolescence. J Pediatr Psychol. 2002 Jun;27(4):373-84.

2. De Genna N, Goldschmidt L, Richardson GA. Prenatal cocaine exposure and age of sexual initiation: direct and indirect effects. Drug Alcohol Depend. 2014 Dec 1;145:194-200.

3. Zimmer-Gembeck MJ, Helfand M. Ten years of longitudinal research on U.S. adolescent sexual behaviour: developmental correlates of sexual intercourse, and the importance of age, gender and ethnic background. Dev Rev. 2008;28(2):153-224.

4. Kaestle CE, Halpern CT, Miller WC, Ford CA. Young age at first sexual intercourse and sexually transmitted infections in adolescents and young adults. Am J Epidemiol. 2005 Apr 15;161(8):774-80.

5. Singh S, Wulf D, Samara R, Cuca Y. Gender differences in the timing of first intercourse: data from 14 countries. Int Fam Plan Perspect. 2000;26(1):21-8

6. Madkour AS, de Looze M, Ma P, Halpern CT, Farhat T, Ter Bogt TF, et al. Macro-level age norms for the timing of sexual initiation and adolescents' early sexual initiation in 17 European countries. J Adolesc Health. 2014 Jul;55(1):114-21.

7. Weiss P, Zvěřina J. Sexual behaviour of the population of the Czech Republic - IV. Prague: DEMA; 2009. (In Czech.)

8. Máthé R, Ritomský A. Specific issues regarding the first sexual intercourse in Slovakia. Sexuológia. 2005;5(1):20-9. (In Slovak.) 
9. Kastbom ÅA, Sydsjö G, Bladh M, Priebe G, Svedin CG. Sexual debut before the age of 14 leads to poorer psychosocial health and risky behaviour in later life. Acta Paediatr. 2015 Jan;104(1):91-100.

10. Johnson AM, Wadsworth J, Wellings K, Field J. Sexual attitudes and lifestyles. Oxford: Blackwell Scientific; 1994.

11. Michael RT, Gagnon JH, Laumann EO, Kolata G. Sex in America: a definite survey. Boston: Little Brown \& Co; 1995.

12. Bingham CR, Miller BC, Adams GR. Correlates of age at first sexual intercourse in a national sample of young women. J Adolesc Res. 1990 Jan;5(1):18-33.

13. Odynak D. Age at first intercourse in Canada: some recent findings. Can Stud Popul. 1994;21(1):51-70.

14. Spira A, Bajos N. Sexual behaviour and AIDS. Aldershot: Avebury; 1994.

15. Safeguarding Teenage Intimate Relationships. STIR findings [Internet]. STIR it App [cited 2015 March 3]. Available from: http://stiritapp.eu/ stir-findings/.

16. Young-Powell A. Students turn to porn for sex education. The Guardian [Internet]. 2015 Jan 29 [cited 2015 March 3]. Available from: http://www. theguardian.com/education/2015/jan/29/students-turn-to-porn-for-sexeducation.

17. Currie C, Inchley J, Molcho M, Lenzi M, Veselska Z, Wild F. Health Behaviour in School-aged Children (HBSC) study protocol: background, methodology and mandatory items for the 2013/14 survey. St Andrews: CAHRU; 2014

18. Wyatt GE. The relationship between child sexual abuse and adolescent sexual functioning in Afro-American and white American women. Ann N Y Acad Sci. 1988;528:111-22.

19. Jeffrey TB, Jeffrey LK. Psychologic aspects of sexual abuse in adolescence. Curr Opin Obstet Gynecol. 1991 Dec;3(6):825-31.

20. Rogers CM, Terry T. Clinical intervention with boy victims of sexual abuse. In: Stuart JR, Geer JG. Victims of sexual aggression. New York: Van Nostrand Reinhold; 1984. p. 91-104.
21. Gianotten W. Sexology in the Netherlands. In: Weiss P, et al. Sexual abuse of children. Prague: Grada Publishing; 2005. (In Czech.)

22. Lukšík I. Interacting of sexual and drug risks: summary of results from tow socio-cultural environments. Cesk Psychol. 2003:47(5):438-52. (In Slovak.)

23. Gibbs N. How should we teach our children about sex? Time Magazine [Internet]. Time; 1993 [cited 2015 March 1]. Available from: http:// content.time.com/time/magazine/article/0,9171,978565,00.html.

24. Wright R. The moral animal: why we are what we are. Prague: NLN; 2002. (In Czech.)

25. Voydanoff P, Donnelly BW. Adolescent sexuality and pregnancy. Newbury Park: Sage Publications; 1990.

26. Larson L, Goltz HW, Hobart C. Families in Canada: social context, continuities and changes. Scarborough: Prentice-Hall International; 1994.

27. Zimmerman RS, Atwood KA. Predicts of first sexual intercourse in the 9th through 11th grades: how do they change over time? In: 128th Annual Meeting of APHA; 2000 Nov 12-16; Boston, USA.

28. Jordahl T, Lohman BJ. A Bioecological analysis of risk and protective factors associated with early sexual intercourse of young adolescents. Child Youth Serv Rev. 2009 Dec 1;31(12):1272-82.

29. Kontula O. Trends in teenage sexual behaviour: pregnancies, sexually transmitted infections and HIV infections in Europe. European Population Papers Series, no. 14. Strasbourg: Council of Europe, the European Population Committee; 2002.

30. Wellings K, Nanchahal K, Macdowall W, McManus S, Erens B, Mercer $\mathrm{CH}$, et al. Sexual behaviour in Britain: early heterosexual experience. Lancet. 2001 Dec 1;358(9296):1843-50.

Received January 19, 2017 Accepted in revised form June 4, 2017 